\title{
JSGE Clinical Practice Guidelines 2014: standards, methods, and process of developing the guidelines
}

\author{
Masahiro Yoshida $\cdot$ Yoshikazu Kinoshita • \\ Mamoru Watanabe · Kentaro Sugano
}

Received: 29 September 2014/ Accepted: 29 October 2014/Published online: 2 December 2014

(C) Springer Japan 2014

\section{Background to and basic policy in the development of the JSGE Clinical Practice Guidelines 2014}

The Japanese Society of Gastroenterology (JSGE) had already published its guidelines on six diseases, namely, gastroesophageal reflux disease (GERD), peptic ulcer disease, liver cirrhosis, Crohn's disease, gallstone disease, and chronic pancreatitis, in Japanese, and distributed them, together with their sister versions for laypeople, to its members. These guidelines are sold in bookstores, widely used even by people who are not JSGE members, and the contents are often cited in other publications. Considering the need and importance of having proper guidelines on the so-called common diseases, JSGE conducted a questionnaire survey on JSGE councilors to collect their views on priority diseases for which additional guidelines should be developed, and decided to prepare additional guidelines for functional gastrointestinal disorder, colorectal polyp, and NAFLD/NASH. In the subsequent process of developing the guidelines, it was further decided that two separate guidelines, one for functional dyspepsia (FD) and the other for irritable bowel syndrome (IBS), should be developed for functional gastrointestinal disorders. Therefore, the second series of JSGE guidelines (JSGE Clinical Practice Guidelines 2014; Japanese version) was published, covering a total of four diseases.

In the first series of the guidelines for the six diseases, JSGE asked related societies to recommend members who

The members of the Working Committee are listed in the Appendix in the text.

M. Yoshida $(\bowtie) \cdot$ Y. Kinoshita $\cdot$ M. Watanabe $\cdot$ K. Sugano Guidelines Committee for the Japanese Society of

Gastroenterology (JSGE), K-18 Building 8F, 8-9-13 Ginza, Chuo, Tokyo 104-0061, Japan

e-mail: dr44da@yahoo.co.jp will make up the guidelines' writing and assessment committees, and asked the recommended persons to join the writing committee. The JSGE Clinical Practice Guidelines 2014 were developed with the strengthened cooperation of societies closely related to each of the covered diseases, with JSGE forming the core. In other words, the guidelines on functional gastrointestinal disorders were developed with the cooperation of the Japanese Gastroenterological Association and the Japanese Society of Neurogastroenterology; the guidelines on colorectal polyp, with the cooperation of the Japanese Gastroenterological Association, the Japanese Society of Gastrointestinal Cancer Screening, Japan Gastroenterological Endoscopy Society, the Japan Society of Coloproctology, and the Japanese Society for Cancer of the Colon and Rectum; and the guidelines on NAFLD/NASH, with the cooperation of the Japan Society of Hepatology. We would like to express our sincere appreciation to these societies. While a wide variety of guidelines are being developed today, it is truly significant to have multiple specialist societies working together, based on shared understanding, to prepare the guidelines for common diseases for the benefit of daily clinical practice. It is hoped that such a collaborative approach by related societies continues to be adopted in the future in an effort to prevent discrepancies between different guidelines.

As in the first series of the guidelines, interim reports and presentation of the final draft guidelines for the second series were made at the JSGE general meetings and annual meetings to promote the exchange of opinions among JSGE members. In addition, the society sought public comments on its website, ensuring transparency and openness of the writing process. However, as for the public comment on the website, the public commenting system was not sufficiently user-friendly, and the commenting period not sufficiently long for canvassing diverse opinions. Even after the 
publication of the guidelines, there is a need to improve the system so that questions, opinions, and new findings can be collected and reflected in the guidelines.

For the level of evidence and strength of recommendations in the JSGE Clinical Practice Guidelines 2014, we decided to adopt systematic concepts advocated by the GRADE (The Grading of Recommendations Assessment, Development and Evaluation) Working Group. The GRADE system does not merely decide the strength of recommendations from available evidence, but also takes into consideration the recommendations' benefits to patients and costs. Even in randomized controlled trials, the system requires the assessment of the level of quality of evidence to be adjusted depending on the methodology used in the trials. Therefore, the system's focus on how the outcomes of clinical interventions and recommendations will benefit patients led us to conclude that the system would be useful in developing guidelines that gave priority to the standpoint of patients. In evidence-based medicine (EBM), developing guidelines based on the GRADE system is now an international norm. On the other hand, the use of the GRADE system in clinical guidelines has hardly ever been tried in Japan before, requiring proper understanding of the system and meticulous handling of literature on our part. We also plan to use the GRADE system in the ongoing work for updating the first series of six guidelines mentioned above, based on the sunset rule (Sunset rule: guidelines in existence for long periods after their initial publication are discarded as their validity can no longer be guaranteed).

As mentioned above, the newly published JSGE guidelines were developed through close collaboration among other related societies in Japan, and contain methodologies and materials serving as standards in clinical gastroenterology in Japan. As such, we decided to distribute them internationally in the form of an English-language paper.

Needless to say, considerable time and energy is needed to produce clinical practice guidelines, but much can be gained from that process. Above all, we hope that these guidelines will raise the level of clinical gastroenterology and contribute to providing proper care for the sake of patients.

Keywords Methodology - Clinical Practice Guidelines 2014 . The Japanese Society of Gastroenterology

\section{Process of developing the guidelines}

Initiation of the development of the JSGE clinical practice guidelines 2014

The JSGE had already published its guidelines on six diseases, namely, gastroesophageal reflux disease (GERD), peptic ulcer disease, liver cirrhosis, Crohn's disease, gallstone disease, and chronic pancreatitis, in Japanese, and distributed them, together with their sister versions for laypeople, to JSGE members. These guidelines are sold in bookstores, widely used even by people who are not JSGE members, and the contents are often cited in other publications.

Considering the need and importance of having proper guidelines on the so-called common diseases, the JSGE Clinical Practice Guidelines Steering Committee (Table 1) conducted, in June 2005, a questionnaire survey on JSGE councilors to collect their views on priority diseases for which additional guidelines should be developed and published. Based on the results of the survey, the committee decided to prepare additional guidelines for functional gastrointestinal disorder, colorectal polyp, and NAFLD/NASH, and to establish the Guidelines Committees. In the subsequent process of developing the guidelines, it was further decided that two separate guidelines, one for functional dyspepsia (FD) and one for irritable bowel syndrome (IBS), should be developed for functional gastrointestinal disorders, bringing the total number of additional guidelines to four.

Based on a project plan and timeline for developing the four guidelines, which were submitted by the Clinical Practice Guidelines Steering Committee, the JSGE secured funding for the project, and the work of preparing the guidelines was started.

Composition of the clinical practice guideline committee

Each Guideline Committee consists of the Chair, the Guideline Writing Committee, and the Guideline Assessment Committee. It should be mentioned that the JSGE Clinical Practice Guidelines 2014 were developed with the strengthened cooperation of societies closely related to each disease, with JSGE forming the core. In other words, the guidelines on functional gastrointestinal disorders were developed with the cooperation of the Japanese Gastroenterological Association and the Japanese Society of Neurogastroenterology; the guidelines on colorectal polyp, with the cooperation of the Japanese Gastroenterological Association, the Japanese Society of Gastrointestinal Cancer Screening, Japan Gastroenterological Endoscopy Society, the Japan Society of Coloproctology, and the Japanese Society for Cancer of the Colon and Rectum; and the guidelines on NAFLD/NASH, with the cooperation of the Japan Society of Hepatology. We would like to express our sincere appreciation to these societies. While a wide variety of guidelines are being developed today, it is truly significant to have multiple specialist societies working together, based on shared understanding, to prepare the guidelines for common diseases for the benefit of daily 
Table 1 JSGE Clinical Practice Guidelines Steering Committee

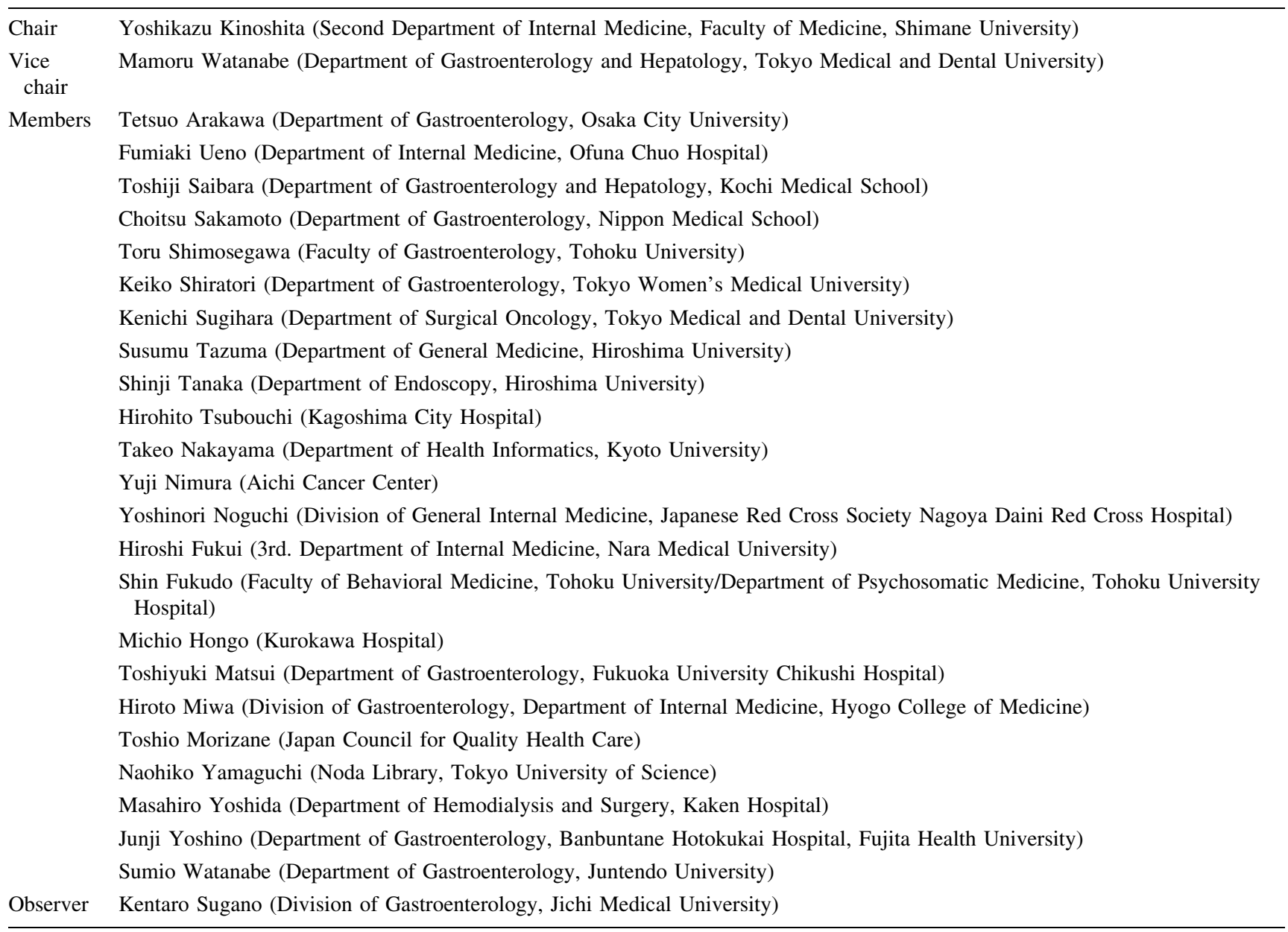

clinical practice. It is hoped that such a collaborative approach by related societies continues to be adopted in the future in an effort to prevent discrepancies between different guidelines.

Process of developing the clinical practice guidelines and universality of their contents

\section{Conflict of interest (COI)}

The members of the JSGE Clinical Practice Guidelines Steering Committee make conflict of interest (COI) disclosure to JSGE's COI Committee every year. All members of the Clinical Practice Guideline Committees for the four types of diseases made their COI disclosure prior to the writing of the guidelines, in an effort to prevent economic-related bias in the contents of the guidelines. Moreover, by building a system of collaboration with multiple related societies as mentioned above, an effort was made to avoid a conflict of scientific interest so as to prevent JSGE from solely promoting its scientific interest.

\section{Economic independence}

The JSGE bore all costs and expenses related to the writing and publication of the four JSGE Clinical Practice Guidelines 2014, without receiving any funding, donations, etc., from pharmaceutical or other companies.

Notes on the use of the guidelines

Although the Guideline Writing Committee and the Guideline Assessment Committee of the JSGE guidelines are responsible for the contents of the guidelines, the attending physician should be responsible for the outcomes of the actual clinical care provided.

Generally speaking, the contents of the JSGE Clinical Practice Guidelines are meant to assist decision-making in 
clinical practice and are not to be used as exhibits in medical practice suits, etc.

Purpose of the JSGE Clinical Practice Guidelines 2014

The purpose of the JSGE Clinical Practice Guidelines 2014 is to develop and publish guidelines on the so-called common diseases that clinicians often encounter in the field of gastroenterology and contribute to providing appropriate care for patients.

\section{Study population (disease)}

The study population (disease) is patients diagnosed with or suspected of having one of the four types of diseases. The laboratory tests, treatment, and instructions on drug dosage and use contained in the guidelines are basically for use in adults and are not applicable to children.

\section{Users}

The users of the guidelines are general clinicians treating lesions of digestive organs. The users should note that the guidelines are meant only as a general guide and that they need to base their decisions cautiously in consideration of individual patients' opinions, age, complications, and social conditions.

Method for writing the clinical practice guidelines

\section{Scoping}

The Guideline Writing Committees for the four Clinical Practice Guidelines began by compiling the clinical and epidemiological characteristics of each disease as well as the healthcare background in Japan (particularly in relation to care provided from medical insurance payments). Based on this compilation, each Writing Committee deliberated on the scope of the guidelines and on the key clinical issues for which recommended care should be presented. The scoping document was approved after review by the Guideline Assessment Committee.

With respect to functional dyspepsia (FD) in particular, the term "functional dyspepsia" was added for insurance payment purposes for the first time in Japan in May 2013. Moreover, the publication of our clinical practice guidelines for this disease helped to attract attention to the disease as well as lay the foundation for providing accurate diagnosis and better treatment for patients with functional gastrointestinal disorders. The guidelines, however, do contain a caveat that as acotiamide is the only drug covered by insurance in Japan for FD today, the treatment recommended in the guidelines has certain limitations in terms of insurance coverage.

In July 2011, the Guidelines Steering Committee deliberated on the basic policy and timeline for the writing of the guidelines, and decided to adopt the concepts of the GRADE system (reference [2-24]) in the writing of the guidelines. The first GRADE system workshop was held in the same month, and the work of drawing up clinical questions (CQs) was started.

\section{Preparation of clinical questions $(C Q)$ and literature search}

Based on the key clinical issues identified in the scoping document, each Guideline Writing Committee prepared the clinical questions (CQs), which were approved after review by the Guideline Assessment Committee.

Starting in November 2011, the Writing Committee extracted keywords from the CQs to search and collect scientific literature from MEDLINE and Cochrane Library databases for English-language literature and from the Japan Medical Abstracts Society (JAMAS) database for Japanese-language literature. The keywords, search formulas, and search periods used for the search are expected to be shown on the JSGE website. The search period was set between 1983 and September 2011. All literature from outside this period was specified as such. Literature that missed the keyword search, but nonetheless needed to be cited in the guidelines, was specified as hand-searched literature.

From the collected literature, the committee selected clinical studies on human subjects and excluded literature on animal experiments or genetic research. The experts' personal opinions that were not based on patient data were used as reference but not as evidence.

\section{Systematic review methodology (assessment of a body of evidence)}

Literature review: preparation of structured abstracts The committee further narrowed down the literature through primary and secondary screening, and created structured abstracts containing study design (Ref. [1]; Table 2) and other information about the selected papers.

The committee rated the risk of bias for RCTs and observational studies (Table 3). It assessed a body of evidence by referring to the concepts of the GRADE system (Ref. [2-21]; Table 4), and determined and recorded the quality of a body of evidence for each CQ (Table 5). 
Table 2 Study designs

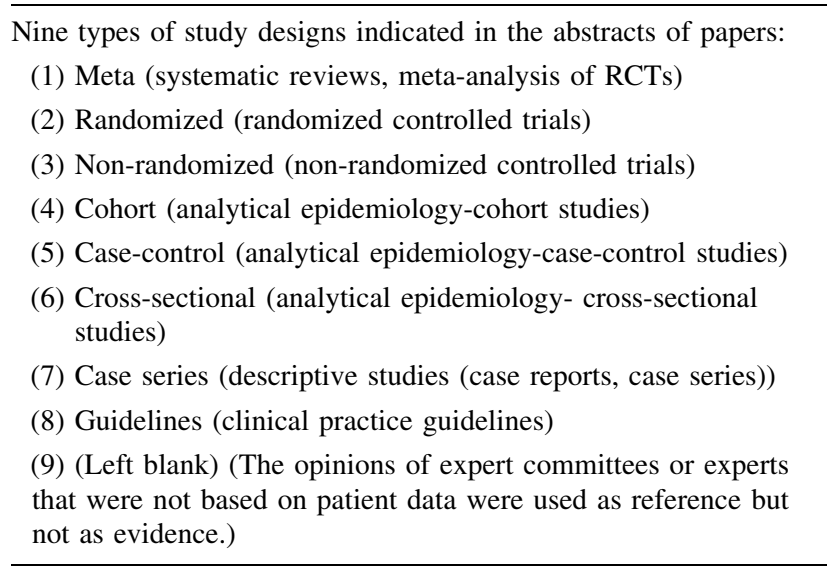

Table 3 Assessment of risk of bias (Compiled from Ref. [13], with modifications)

\section{Selection bias}

\section{(1) Random sequence generation}

Is there a detailed description on the randomization of patient allocation?

(2) Concealment

Is the allocation of patients concealed to the individuals allocating the patients?

Performance bias

(3) Blinding

Are the study subjects blinded? Are the care providers blinded? Detection bias

(4) Blinding

Are the individuals assessing the outcome blinded?

Attrition bias

(5) ITT analysis

Is the intention-to-treat (ITT) principle employed and adhered to with regard to drop-outs in the follow-up?

(6) Outcome reporting bias

Are the data on each of the main outcomes fully reported (including data adopted or excluded from analysis)?

(7) Other biases

Selective outcome reporting: Are there outcomes, even though included in the research design, that are unreported?

Stopping early: Is the trial stopped early for apparent benefit?

Others

Method for defining the quality of evidence The same four categories were used for rating the quality of evidence for both papers from abroad and Japan. To prepare recommendations for CQs, the committee identified outcomes for each CQ, evaluated the benefits and harms, and rated the quality of a body of evidence across multiple literature using the quality categories identified in Table $4 a-d)$.
Table 4 Comprehensive rating of collected multiple literature by outcome and study design (compiled from Ref. [12], with modifications)

(a) Initial assessment: Rating of each study design group

Meta and Randomized: initial rating of "A"

Non-randomized, Cohort, Case-control, and Cross-sectional: initial rating of " $\mathrm{C}$ "

Case series: initial rating of " $\mathrm{D}$ "

(b) Reasons to rate down the quality of evidence

There is risk of bias pertaining to the quality of the study

There is inconsistency of results

There is indirectness of evidence

There is imprecision of data

There is a strong likelihood for publication bias

(c) Reasons to rate up the quality of evidence

There is large magnitude of effect and no confounders

There is dose-response gradient

Plausible confounding results in underestimating apparent effect

(d) Comprehensive rating: The final quality of evidence in rated in accordance with four categories of A, B, C, or D

Table 5 Quality of evidence (compiled from Ref. [12], with modifications)

(A) Quality of evidence-high

We are very confident that the true effect lies close to that of the estimate of the effect

(B) Quality of evidence-moderate

We are moderately confident in the effect estimate:

The true effect is likely to be close to the estimate of the effect, but there is a possibility that it is substantially different.

(C) Quality of evidence-low

Our confidence in the effect estimate is limited:

The true effect may be substantially different from the estimate of the effect

(D) Quality of evidence-very low

We have very little confidence in the effect estimate:

The true effect is likely to be substantially different from the estimate of effect

As for the insurance coverage for each of the evidence presented in the guidelines, it was mentioned in the body of the text of the guidelines only if there was no insurance coverage.

\section{Determination of the strength of recommendations}

Based on the results obtained from the above work, the committee wrote and presented the draft recommendations for treatment. It then organized a consensus meeting to decide on the strength of each recommendation. 
Table 6 Strength of recommendation (compiled from Ref. [23], with modifications)

Strength of recommendation 1 (strong recommendation)

We recommend conducting...

We recommend not to conduct...

Strength of recommendation 2 (weak recommendation)

We suggest conducting...

We suggest not to conduct...

The strength of each recommendation was rated based on the four criteria of (1) strength of the evidence, (2) patients' preferences, (3) benefits and harms, and (4) cost assessment. The meeting participants voted based on the Delphi method and nominal group technique (NGT), and the consensus was deemed to have been reached with $70 \%$ or more in agreement. If a consensus was not reached after the first round of voting, they participated in the successive iterations of voting, following announcement of the results in the first voting round and discussions that also considered the state of healthcare in Japan. The committee made a synthetic assessment of the results to determine the strength of each recommendation (Table 6) and indicated it the box provided in the guidelines.

The strength of a recommendation was indicated as either " 1 " (strong recommendation) or " 2 " (weak recommendation). Because the expressions "recommend strongly" and "recommend weakly" did not sit well in the text of the guidelines, the committee used the expressions shown in Table 6.

\section{Public comment}

The draft guidelines were made public on the JSGE website in December 2013 for public comment. The comments were used to make the final revisions to the draft.

\section{Implementation}

The four JSGE Clinical Practice Guidelines 2014 were distributed to JSGE-accredited institutions and other related institutions. The guidelines are also available on the JSGE website, and can also be purchased as publications from stores.

Updating the JSGE clinical practice guidelines 2014

JSGE plans to update the guidelines at an appropriate time, with the JSGE Guideline Committee playing the central role in the task.

Acknowledgments We would like to express our respect to all members of the Guideline Writing Committees and the Guideline
Assessment Committees of the Clinical Practice Guideline Committees (for functional dyspepsia, irritable bowel syndrome, colorectal polyp, and NAFLD/NASH).

Conflict of interest The authors declare that they have no conflicts of interest.

\section{Appendix}

Committee members: Masahiro Yoshida (Department of Hemodialysis and Surgery, Kaken Hospital), Yoshikazu Kinoshita (Second Department of Internal Medicine, Faculty of Medicine, Shimane University), Mamoru Watanabe (Department of Gastroenterology and Hepatology, Tokyo Medical and Dental University), Kentaro Sugano (Division of Gastroenterology, Jichi Medical University).

\section{Reference}

1. Fukui T, Yamaguchi N, Morizane T, Yoshida M, Kojimahara N, editors. MINDS handbook for clinical practice guideline development 2014. Tokyo: Igaku-shoin; 2014.

2. Aihara M, Mihara H, Murayama T, Aihara T, Fukuda S. GRADE System for clinical practice guideline-therapeutic intervention. Hirosaki: Toppan-media; 2010.

3. The GRADE* working group. Grading quality of evidence and strength of recommendations. BMJ. 2004;328:1490-4 (printed, abridged version).

4. Guyatt GH, Oxman AD, Vist G, GRADE Working Group, et al. Rating quality of evidence and strength of recommendations GRADE: an emerging consensus on rating quality of evidence and strength of recommendations. BMJ. 2008;336:924-6.

5. Guyatt GH, Oxman AD, Kunz R, GRADE Working Group, et al. Rating quality of evidence and strength of recommendations: what is "quality of evidence" and why is it important to clinicians? BMJ. 2008;336:995-8.

6. Schünemann HJ, Oxman AD, Brozek J, GRADE Working Group, et al. Grading quality of evidence and strength of recommendations for diagnostic tests and strategies. BMJ. 2008;336:1106-10.

7. Guyatt GH, Oxman AD, Kunz R, GRADE working group, et al. Rating quality of evidence and strength of recommendations: incorporating considerations of resources use into grading recommendations. BMJ. 2008;336:1170-3.

8. Guyatt GH, Oxman AD, Kunz R, GRADE Working Group, et al. Rating quality of evidence and strength of recommendations: going from evidence to recommendations. BMJ. 2008;336:1049-51.

9. Jaeschke R, Guyatt GH, Dellinger P, GRADE working group, et al. Use of GRADE grid to reach decisions on clinical practice guidelines when consensus is elusive. BMJ. 2008;337:a744.

10. Guyatt G, Oxman AD, Akl E, et al. GRADE guidelines 1 . Introduction-GRADE evidence profiles and summary of findings tables. J Clin Epidemiol. 2011;64:383-94.

11. Guyatt GH, Oxman AD, Kunz R, et al. GRADE guidelines 2. Framing the question and deciding on important outcomes. J Clin Epidemiol. 2011;64:295-400.

12. Balshem H, Helfand M, Schunemann HJ, et al. GRADE guidelines 3: rating the quality of evidence. J Clin Epidemiol. 2011;64:401-6. 
13. Guyatt GH, Oxman AD, Vist G, et al. GRADE guidelines 4: rating the quality of evidence-study limitation (risk of bias). J Clin Epidemiol. 2011;64:407-15.

14. Guyatt GH, Oxman AD, Montori V, et al. GRADE guidelines 5: rating the quality of evidence-publication bias. J Clin Epidemiol. 2011;64:1277-82.

15. Guyatt G, Oxman AD, Kunz R, et al. GRADE guidelines 6 . Rating the quality of evidence-imprecision. J Clin Epidemiol. 2011;64:1283-93.

16. Guyatt GH, Oxman AD, Kunz R, The GRADE Working Group, et al. GRADE guidelines: 7. Rating the quality of evidenceinconsistency. J Clin Epidemiol. 2011;64:1294-302.

17. Guyatt GH, Oxman AD, Kunz R, The GRADE Working Group, et al. GRADE guidelines: 8. Rating the quality of evidenceindirectness. J Clin Epidemiol. 2011;64:1303-10.

18. Guyatt GH, Oxman AD, Sultan S, The GRADE Working Group, et al. GRADE guidelines: 9. Rating up the quality of evidence. J Clin Epidemiol. 2011;64:1311-6.

19. Brunetti M, Shemilt I, The GRADE Working, et al. GRADE guidelines: 10. Considering resource use and rating the quality of economic evidence. J Clin Epidemiol. 2013;66:140-50.
20. Guyatt G, Oxman AD, Sultan S, et al. GRADE guidelines: 11 . Making an overall rating of confidence in effect estimates for a single outcome and for all outcomes. J Clin Epidemiol. 2013;66:151-7.

21. Guyatt GH, Oxman AD, Santesso N, et al. GRADE guidelines 12. Preparing summary of findings tables binary outcomes. J Clin Epidemiol. 2013;66:158-72.

22. Thorlund K, Oxman AD, Walter SD, et al. GRADE guidelines 13. Preparing summary of findings tables-continuous outcomes. J Clin Epidemiol. 2013;66(2):173-83.

23. Andrews J, Guyatt G, Oxman AD, et al. GRADE guidelines: 14 . Going from evidence to recommendations: the significance and presentation of recommendations. JCE. 2013;66(2):719-25.

24. Andrews JC, Schunemann HJ, Oxman AD, et al. GRADE guidelines: 15. Going from evidence to recommendation-determinants of a recommendation's direction and strength. J Clin Epidemiol. 2013;66(7):726-35. 